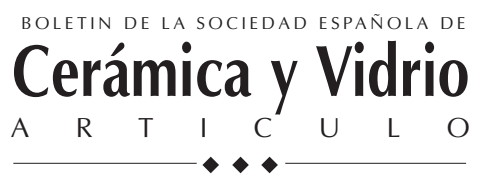

\title{
Síntesis de Wollastonita por reacción en estado sólido a partir de Diatomitas Españolas. Estudio Cinético
}

\author{
F. SANDOVAL y A. IBÁNEZ \\ Instituto de Cerámica y Vidrio. Departamento de Cerámica, C.S.I.C., Ctra. Madrid-Valencia, Km. 24,300. 28500 - Arganda del Rey (Madrid).
}

\begin{abstract}
Se estudia la síntesis de wollastonita por reacción en estado sólido a partir de dos diatomitas españolas. Los resultados obtenidos con estas dos materias primas se comparan con los obtenidos con un cuarzo y un gel de sílice, de distinta superficie específica, para observar la influencia de esta propiedad física en la reacción de síntesis. Se estudian por otra parte, el mecanismo y la cinética de la reacción. Con respecto a esta última, se aplica el modelo matemático de Jander, que permite describir la fase inicial del proceso. Posteriormente la reacción avanza más lentamente, y la mencionada ecuación no es aplicable a la totalidad del proceso, por lo cual, se hace necesario buscar otro modelo, que explique mejor la cinética de la reacción. Dicho modelo no es otro que el de Avrami.
\end{abstract}

Palabras clave: velocidad de reacción, diatomita, $\beta$-silicato dicálcico y wollastonita.

\section{Synthesis of wollastonite by solid state reaction from Spanish diatomites. Kinetic study}

The synthesis of wollastonite by the solid state reaction technique from two Spanish diatomites is studied. The results obtained were compared with those of a quartz and a silica gel of different specific surface area, with in order to verify the effect of the physical property on the reaction of synthesis. The mechanism and kinetic of the reaction were also studied. With respect to he kinetic the mathematical model of Jander was applied. At the early stage of the reaction the model is applicable. However, subsequently, the reaction becomes slower and the the model of Jander does not explain the kinetic of the whole process. Thus, it is necessary to look for other model, which explains better the reaction of synthesis. That of Avrami gets the objetive.

Key words: reaction rate, diatomite, $\beta$-dicalcium silicate, wollastonite

\section{INTRODUCCIÓN}

Durante muchos años se ha venido prestando atención a los silicatos y silicoaluminatos de calcio y/o magnesio, por las propiedades que conferían a las pastas en cuya composición intervenían, a las que hacían aptas para procesos de cocción rápida (1). En concreto, la wollastonita $\left(\mathrm{B}_{-}-\mathrm{CaSiO}_{3}\right)$ ha recibido mucha atención durante la pasada década. El mineral comenzó a utilizarse en la industria cerámica de revestimiento de EE.UU., en los años 40 (2). No obstante, la explotación del mineral a escala industrial comenzó en los 50, siendo sus principales productores: EE.UU., India y Méjico. La producción americana alcanzó la cota máxima del mercado mundial, con el $85 \%$ del mismo (3).

El nombre de wollastonita procede del químico y mineralólogo inglés $\mathrm{W}$. $\mathrm{H}$. Wollaston (1776 - 1828). La fase $\mathrm{B}_{-} \mathrm{CaSiO}_{3}$ es estable a baja temperatura, y se transforma en pseudowollastonita a $1125^{\circ} \mathrm{C}$. Existen varios polimorfos de baja temperatura. El más común es la wollastonita-1T, de simetría triclínica; mucho más rara es la wollastonita-2M, de simetría monoclínica. La Comission New Minerals and Nomenclature, de la Industrial Mineral Association, usa el término wollastonita para referirse a la forma de baja temperatura del mineral (4).

La producción de wollastonita se ha doblado entre 1986 y 1995, con un crecimiento medio anual del $10 \%$. Se estima que esta tasa de crecimiento continuará al menos hasta el 2000, debido a la extensión en las aplicaciones finales de la wollastonita, principalmente en plásticos. En la actualidad el mercado de wollastonita ha crecido de manera explosiva hasta alcanzar una producción mundial estimada en 500.000 tn/ año (5). Estados Unidos y China producen el $75 \%$ de este total mundial. Los otros países que producen wollastonita de un modo significativo, son Finlandia (28.000 tn./año) y la India (80.000 tn/año). Las nuevas explotaciones desarrolladas en Méjico y Canadá han convertido a estos países en importantes productores desde 1997, con un potencial conjunto de producción cercano a las 250.000 tn/ año.

El principal uso de la wollastonita se centra en la cerámica, a la que se destina un $40 \%$ de la producción total, principalmente para fritas y esmaltes. También se emplea como carga en pinturas anticorrosión, en metalurgia y como sustituto de los asbestos, por su carácter no carcinógeno. Pero donde la demanda de wollastonita ha experimentado un mayor crecimiento ha sido en el campo de los plásticos y de las resinas en el que ya alcanza el $25 \%$ de la demanda total, con tendencia a seguir creciendo (5).

La existencia del mineral en Europa era tan esporádica, que estaba justificada su síntesis, debido a su alto coste de importación. Ante esta situación, a mediados de los setenta comenzó a sintetizarse wollastonita a partir de materias primas locales 
TABLA I.

COMPOSICIONES FORMULADAS

\begin{tabular}{||c|c|c|c|c|c||}
\hline Composicion & $\begin{array}{c}\text { Diatomita } \\
\text { D-1 }\end{array}$ & $\begin{array}{c}\text { Diatomita } \\
\text { D-2 }\end{array}$ & Cuarzo & Gel de sílice & Creta \\
\hline AS & 40,8 & -- & -- & -- & 59,2 \\
\hline BS & -- & 78,5 & -- & -- & 21,5 \\
\hline CS & -- & -- & 37,5 & -- & 62,5 \\
\hline DS & -- & -- & -- & 37,5 & 62,5 \\
\hline
\end{tabular}

abundantes y baratas. En la República Federal de Alemania, en concreto, se disponía para este fin de grandes reservas de caliza y cuarzo (6). La wollastonita sintética poseía unas características tecnológicas bien definidas y reproducibles para su aplicación en cerámica.

La síntesis del mineral vino precedida por investigaciones teóricas sobre:

a) composición química de las materias primas primas de partida.

b) tamaño de partícula óptimo de las mismas.

c) relación molar $\mathrm{CaO} / \mathrm{SiO}_{2}$ óptima.

d) cinética de formación de wollastonita, etc. (7).

Ibañez y Sandoval en un trabajo previo (8) exponen con todo lujo de detalles los distintos procedimientos para sintetizar wollastonita. De entre estos procedimientos, el de reacción en estado sólido a partir de mezclas de $\mathrm{CaCO}_{3}$ y $\mathrm{SiO}_{2}$, resultó ser el más simple. No obstante, este procedimiento estaba condicionado por el tamaño de partícula que debían alcanzar las materias primas, según indicaba Schmalzried (9). El procedimiento era económicamente inviable por los elevados costes de producción debidos al gran consumo energético necesario para moler el cuarzo hasta que éste poseyera una reactividad apropiada. El procedimiento fue notablemente mejorado mediante el empleo de materias primas que aportaban sílice amorfa muy reactiva, como gel de sílice (7), trípoli (10) o utilizando mineralizadores adecuados (11 - 19).

La diatomita $\left(\mathrm{SiO}_{2} \cdot \mathrm{nH}_{2} \mathrm{O}\right)$ constituye una importante variedad de sílice amorfa muy reactiva. La diatomita es un material sedimentario, formado principalmente por esqueletos de dia-

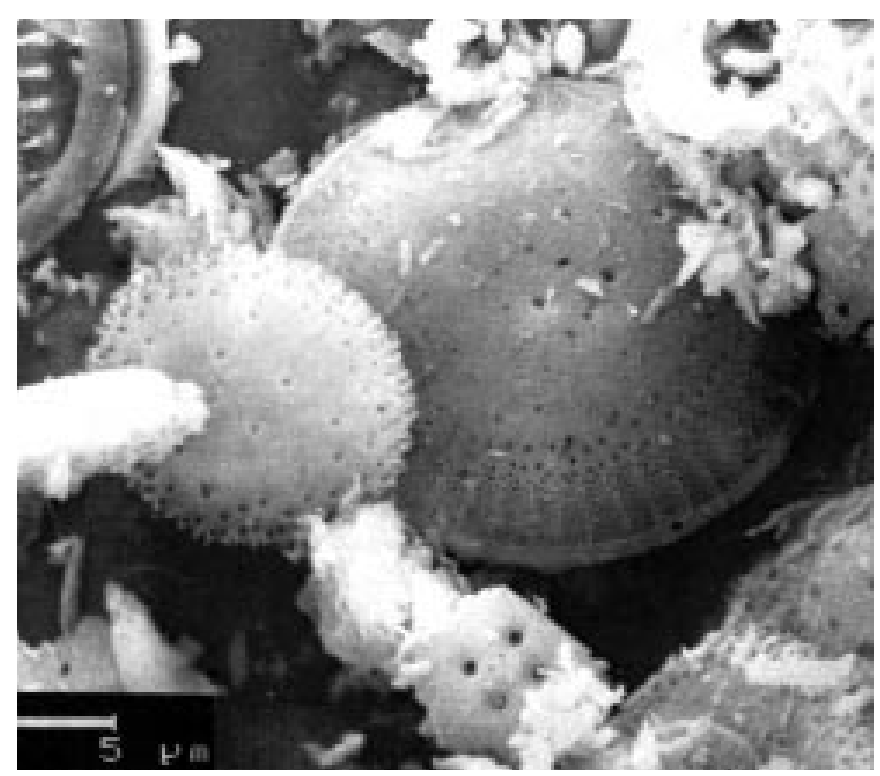

Fig. 1.- Micrografía obtenida por MEB de la diatomita D-2. tomeas compuestos por sílice amorfa (20). El material consiste en partículas de tamaño comprendido entre 10 y $200 \mathrm{~mm}$, conteniendo su estructura entre 80 y $90 \%$ de espacios vacíos (21). En la Fig. 1, se puede apreciar este hecho, donde se muestra una micrografía obtenida por microscopía electrónica de barrido en una diatomita utilizada en el presente trabajo.

El objetivo de éste es estudiar el mecanismo de la reacción de síntesis de wollastonita a partir de dos diatomitas españolas. Los resultados del estudio se comparan con los obtenidos a partir de un cuarzo y un gel de sílice, para verificar la influencia de la superficie específica de las materias primas silíceas en el rendimiento de la reacción. Se estudia asimismo la cinética de la reacción de síntesis, mediante la aplicación de modelos teóricos.

\section{MATERIAS PRIMAS}

Se utilizaron en la investigación las siguientes materias primas:

Diatomitas D-1 (John Manville, S.A.) y D-2, procedente de un depósito localizado entre las provincias de Murcia y Albacete, cuarzo Sibelco SE-6 (Sibelco Española, S.A., Barcelona), gel de sílice Cab-O-Sil (EH-M) (Cabot Corporation, Boston MA, EE.UU.) y creta T-50, procedente de un yacimiento situado en la provincia de Tarragona.

Excepto el gel de sílice, las restantes materias primas fueron suministradas por el Instituto de Tecnología Cerámica (A.I.C.E.), Castellón.

\section{COMPOSICIONES}

Con las materias primas relacionadas más arriba se formularon las composiciones con relación molar $\mathrm{CaO}: \mathrm{SiO}_{2}=1: 1$ que aparecen en la Tabla I.

\section{EXPERIMENTAL}

\subsection{Procesamiento}

Las composiciones se molturaron y homogeneizaron en alcohol isopropílico, en tarros de ágata, con bolas del mismo material, en molino planetario hasta que el rechazo en tamiz de $63 \mu \mathrm{m}$. fue de aproximadamente el 3\%. en peso. El polvo obtenido, desecado, se humectó con 5,5\% de agua y se prensaron uniaxialmente probetas de $20 \mathrm{~mm}$ de espesor y unos $4 \mathrm{~mm}$ de espesor, a $30 \mathrm{MPa}$. Según Kadey (22), esta presión no afecta ni al área superficial de la diatomita, ni a su porosidad interna, tan sólo disminuye su porosidad intraparticular. Las composiciones así procesadas se trataron a $1100{ }^{\circ} \mathrm{C}$ durante espacios de tiempo comprendidos entre $1 / 2$ y $24 \mathrm{~h}$., con una velocidad de calentamiento de $20^{\circ} \mathrm{C} / \mathrm{min}$. Para elegir esta temperatura de tratamiento se ha tenido en cuenta que a $1125^{\circ} \mathrm{C}$ tiene lugar la transformación:

$$
\text { Wollastonita } \rightarrow \text { Seudowollastonita }
$$

\subsection{TECNICAS EXPERIMENTALES}

Se utilizaron las siguientes técnicas experimentales: Análisis químico, granulometría y superficie específica en las 
materias primas silíceas; análisis cuantitativo de fases por difracción de rayos $\mathrm{X}$ mediante el método de eliminación de la matriz de Chung (23), utilizando $\mathrm{CaF}_{2}$ (reactivo Merck, grado de análisis) como patrón interno y finalmente microscopía electrónica de barrido.

\section{RESULTADOS Y DISCUSIÓN}

\subsection{Estudios realizados en las materias primas}

En la Tabla II, se encuentra la composición química de las materias primas utilizadas. La superficie específica y la granulometría de las materias primas silíceas, se encuentran en la Tabla III.

\subsection{Síntesis de wollastonita}

\subsubsection{SEGUIMIENTO DE LA REACCIÓN}

Las curvas de ATD de las composiciones estudiadas se han representado en la Fig. 2. En todas ellas se aprecia un pequeño efecto exotérmico entre 350 y $400^{\circ} \mathrm{C}$, que puede corresponder al comienzo de la formación del $\beta_{-} \mathrm{Ca}_{2} \mathrm{SiO}_{4}\left(\mathrm{~B}_{-} \mathrm{C}_{2} \mathrm{~S}\right.$ : $\mathrm{C}=\mathrm{CaO}, \mathrm{S}$ $=\mathrm{SiO}_{2}$ ); un efecto endotérmico intenso entre 850 y $900^{\circ} \mathrm{C}$, correspondiente a la descomposición del carbonato cálcico y otro exotérmico en el intervalo $900-1000^{\circ} \mathrm{C}$, debido a la cristalización de la wollastonita. En la curva de la composición CS se aprecia además un pequeño efecto endotérmico hacia los $570^{\circ} \mathrm{C}$, que corresponde a la transformación $\alpha \rightarrow B$ del cuarzo.

El estudio de los difractogramas de los productos obtenidos confirma lo expuesto en el párrafo anterior. Así, la primera fase identificada mediante esta técnica fue el $\left(\mathrm{B}_{\mathrm{B}} \mathrm{C}_{2} \mathrm{~S}\right)$ identificado en la literatura como compuesto inicial de las reacciones entre el óxido de calcio y la sílice, en un amplio rango de composiciones (24). El $B-C_{2} S$ presenta dos polimorfos de baja temperatura: $\beta$ y $\gamma$. El polimorfo $\gamma$ cristaliza en el sistema ortorrómbico, posee una estructura relativamente "abierta", de tipo olivino y es estable termodinámicamente a temperatura ambiente, mientras que, el $B$ es monoclínico, más denso y metaestable a temperatura ambiente. La conversión $B \rightarrow \gamma$ puede iniciarse por choque mecánico o presión y una vez iniciada, tiende a ser autocatalítica.

Persiste todavía en la literatura cierta confusión en torno a las transformaciones de fase del $\mathrm{B}-\mathrm{C}_{2} \mathrm{~S}$ al aumentar la temperatura. El esquema generalmente aceptado es el propuesto por Nielsen (25), sin embargo ha sido recientemente mejorado por Remy et al. (26). En las reacciones de transformación del $\aleph_{-} \mathrm{C}_{2} \mathrm{~S}$ hay muchos factores que influyen notablemente en su secuencia de inversiones, tales como, tamaño de grano, perfección cristalina, impurezas, etc., a pesar de tener escasa contribución a la energía libre total. En concreto el efecto de las impurezas es muy notable.

\subsubsection{EVOLUCIÓN DEL CONTENIDO DE $\Re_{-} \mathrm{C}_{2} \mathrm{~S}$}

Aunque según ya se ha indicado, el polimorfo $B$ no es estable termodinámicamente a temperatura ambiente, fué la primera fase observada en las composiciones estudiadas. Este hecho se explica en la literatura (2), por las diferentes densidades aparentes del óxido de calcio y del cuarzo, las cuales originan unas condiciones para el contacto entre partículas pro-
TABLA II.

COMPOSICIÓN QUÚMICA DE LAS MATERIAS PRIMAS

\begin{tabular}{||c|c|c|c|c||}
\hline Oxido & Diatomita D-1 & Diatomita D-2 & Cuarzo & Creta \\
\hline $\mathrm{SiO}_{2}$ & 86,11 & 45,47 & 99,32 & 0,22 \\
\hline $\mathrm{Fe}_{2} \mathrm{O}_{3}$ & 1,12 & 0,50 & 0,34 & 0,02 \\
\hline $\mathrm{TiO}_{2}$ & Ind. & -- & Ind. & -- \\
\hline $\mathrm{Al}_{2} \mathrm{O}_{3}$ & 4,49 & 1,48 & 0,47 & 0,20 \\
\hline $\mathrm{MgO}$ & 0,81 & 0,60 & 0,01 & -- \\
\hline $\mathrm{CaO}$ & 0,57 & 27,37 & 0,05 & 55,26 \\
\hline $\mathrm{Na}_{2} \mathrm{O}$ & 5,05 & 0,08 & 0,04 & -- \\
\hline $\mathrm{K}_{2} \mathrm{O}$ & 0,59 & 0,13 & 0.07 & -- \\
\hline perd. calc. & 0,56 & 23,76 & -- & 43,40 \\
\hline
\end{tabular}

TABLA III.

SUPERFICIE ESPECÍFICA Y GRANULOMETRÍA DE LAS MATERIAS PRIMAS SILÍCEAS

\begin{tabular}{||c|c|c|c|c||}
\hline Determinación & Diatomita D-1 & Diatomita D-2 & Cuarzo & Gel de sílice \\
\hline $\begin{array}{c}\text { Sup. espec. } \\
\left(\mathrm{m}^{2} / \mathrm{g}\right)\end{array}$ & 2,5 & 18,6 & 0,8 & 290 \\
\hline $60-10(\mu \mathrm{m})$ & 22 & 12 & 72 & -- \\
\hline $10-2(\mu \mathrm{m})$ & 70 & 39 & 22 & -- \\
\hline $2-1(\mu \mathrm{m})$ & 6 & 20 & 4 & -- \\
\hline$<1(\mu \mathrm{m})$ & 2 & 29 & 2 & -- \\
\hline Total & 100 & 100 & 100 & -- \\
\hline
\end{tabular}

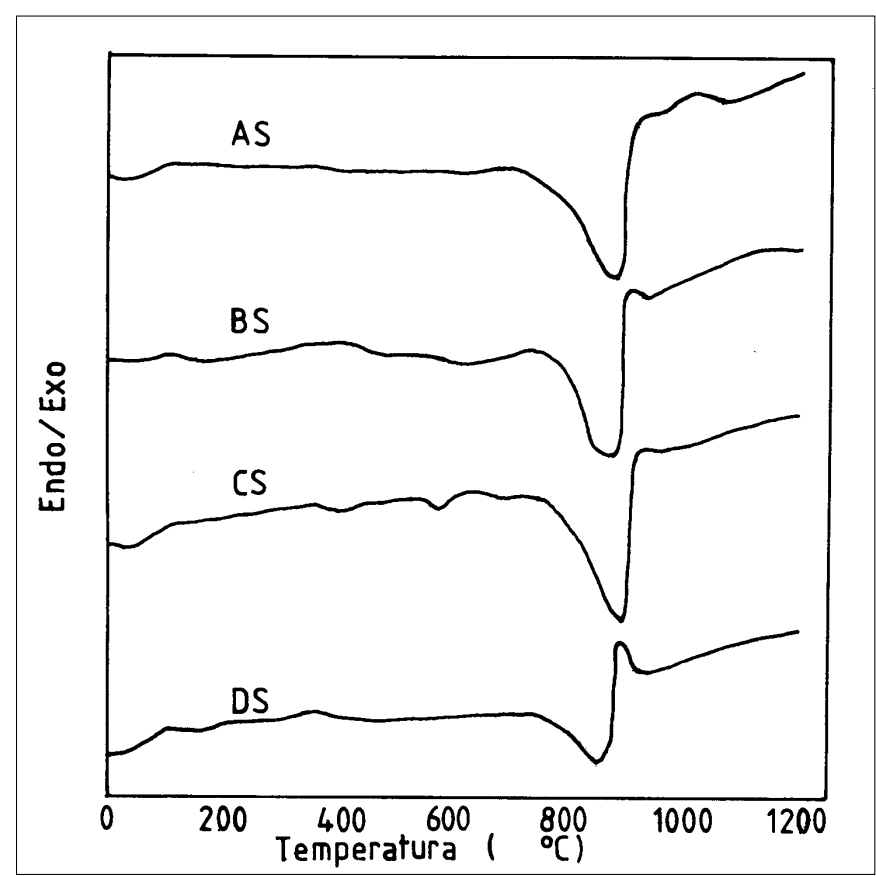

Fig. 2.- Curvas de ATD de las composiciones estudiadas 
pias de una composición rica en $\mathrm{CaO}$, e incluso para una relación $\mathrm{CaO} / \mathrm{SiO}_{2}$ equimolecular. Así, aunque la fase $\gamma$ es termodinámicamente estable a temperatura ambiente, la cinética de la reacción favorece la formación de $\beta-C_{2} S(24)$.

En la Fig. 2, se muestra la evolución del contenido de $\beta_{-} \mathrm{C}_{2} \mathrm{~S}$ de los productos obtenidos con el tiempo de tratamiento. Inicialmente, se observa en esta figura, que la velocidad de formación del compuesto sigue la secuencia:

$$
\text { DS }>\text { BS }>\text { AS }>\text { CS }
$$

Se puede apreciar que esta secuencia es análoga a la de la superficie específica de las materias primas silíceas (Tabla III) presentes en las composiciones de partida. Sin embargo, al aumentar el tiempo de tratamiento, el comportamiento es aparentemente anómalo, ya que la progresiva desaparición del ß$\mathrm{C}_{2} \mathrm{~S}$ por transformación en nuevas fases sigue la secuencia contraria:

$$
\text { AS }>\text { BS }>\text { DS }
$$

es decir, el $\aleph_{-} \mathrm{C}_{2} \mathrm{~S}$ desaparece más lentamente cuanto mayor es la superficie específica de las materias primas mencionadas. Este hecho se puede comprobar en la Fig. 3, donde se representa la proporción de wollastonita formada frente al tiempo de tratamiento. Se puede observar que tras $24 \mathrm{~h}$ de mantenimiento a la temperatura máxima, la fase mencionada se mantiene relativamente estabilizada en el producto DS, mientras que ha desaparecido prácticamente de las composiciones AS y BS y se está formando todavía en la composición CS. La explicación de este comportamiento pasa por la posible estabilización del polimorfo metaestable $\mathrm{B}_{-} \mathrm{C}_{2} \mathrm{~S}$. Hay que tener en cuenta que la transformación $\beta \rightarrow \gamma$ plantea ciertas exigencias estéricas, debido a que la fase $\gamma$ es más voluminosa que la $ß$, por lo que la transformación va acompañada de incremento de volumen, que según Chang y Kriven (27) alcanza el 12\% aproximadamente.

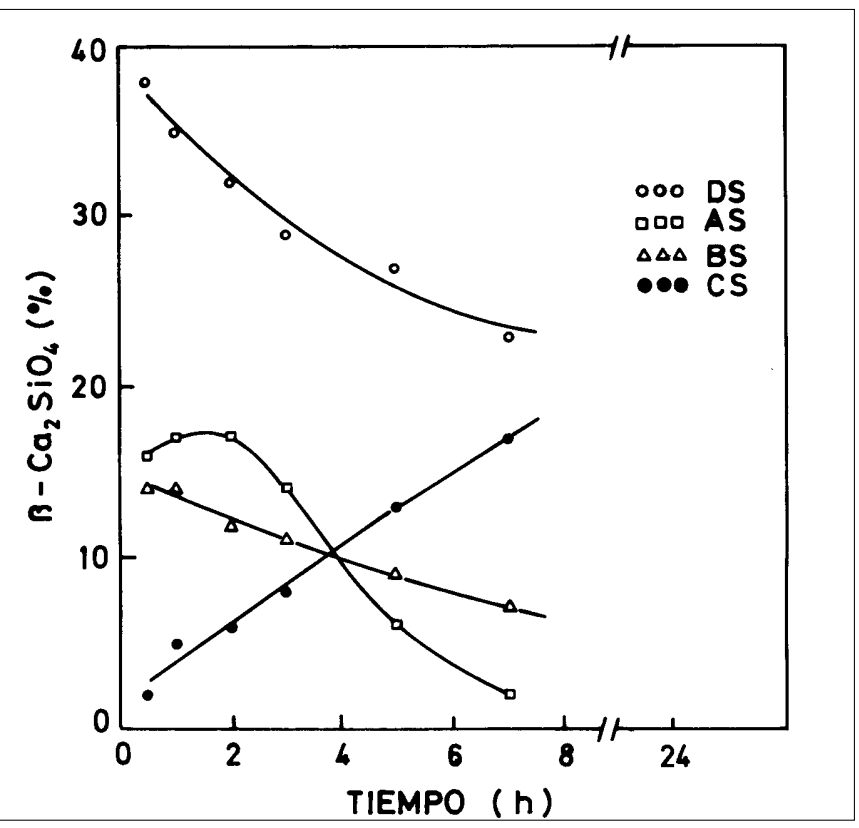

Fig. 3.- Variación del contenido de $\mathrm{B}_{-} \mathrm{C}_{2} \mathrm{~S}$ de las composiciones con el tiempo de tratamiento
En las primeras etapas de la reacción se forma, como se ha indicado, $\mathrm{B}_{-} \mathrm{C}_{2} \mathrm{~S}$, comenzando éste a aparecer alrededor de $350^{\circ} \mathrm{C}$. La proporción de esta fase aumenta a lo largo del tratamiento y evoluciona a la $\gamma$ en pequeñas zonas de reacción donde no tiene limitaciones estéricas. En el caso de que se forme una gran cantidad de fase vítrea, como consecuencia de la gran reactividad de las diatomitas, así como del gel de sílice, los granos de $\mathrm{B}_{-} \mathrm{C}_{2} \mathrm{~S}$ pueden quedar rodeados por esa fase vítrea, la cual constreñiría las transformaciones de aquella fase impidiendo su transformación en el otro polimorfo. Esto explica que la desaparición del $\mathrm{B}_{-} \mathrm{C}_{2} \mathrm{~S}$ sea más lenta cuanto mayor sea la superficie específica de la materia prima silícea. Este mecanismo de estabilización del $\mathrm{B}_{-} \mathrm{C}_{2} \mathrm{~S}$ ya fue sugerido por los autores de este trabajo en uno previo (18) y confirmado posteriormente por Chang y Kriven (27).

Por el contrario, en la composición CS (la cual contiene cuarzo) son las partículas de $\mathrm{CaO}$ las que rodean a las de sílice, que son de mayor tamaño, y la fases $\mathrm{B}_{-} \mathrm{C}_{2} \mathrm{~S}$ no se estabiliza. El incremento de volumen al que anteriormente se ha aludido puede dar lugar en los productos finales a grietas, pudiendo llegarse incluso a la pulverización de las muestras (dusting).

\subsubsection{ESTUDIO CINÉTICO}

\section{i) Modelo de Jander}

Según West (28), las reacciones en estado sólido implican transporte de reactivos a través de los límites de fase o a través del producto formado. El estudio cinético de estas reacciones (en estado sólido) se ha centrado en reactivos policristalinos, y debería esperarse que la velocidad de reacción fuese una función muy compleja, dado el gran número de variables a considerar: distribución de tamaño de las partículas, superficie de contacto entre los reactivos, densidad, porosidad, etc., que en la mayoría de los casos o no permanecen constantes o no se pueden controlar a lo largo de la reacción. Otro aspecto característico de las reacciones en estado sólido es el proceso de formación y crecimiento de núcleos cristalinos de la fase producto. Sin embargo, cuando la etapa controladora del proceso es el transporte de material, muchas reacciones en estado sólido siguen la ley parabólica de velocidad de Jander, especialmente en las primeras etapas de la reacción.

De acuerdo con el mecanismo de reacción propuesto en el apartado anterior, las reacciones principales que tienen lugar son las siguientes:

$$
\begin{gathered}
2 \mathrm{CaO}+2 \mathrm{SiO}_{2} \rightarrow \mathrm{Ca}_{2} \mathrm{SiO}_{4} \text { [2] } \\
\mathrm{Ca}_{2} \mathrm{SiO}_{4} \rightarrow \mathrm{CaSiO}_{3} \text { [3] }
\end{gathered}
$$

Dichas reacciones implicarían el transporte de los reactivos a través de los límites de fase y a través del producto formado. Pero mientras el transporte a través del producto de la reacción es esencialmente un fenómeno de difusión, el transporte en el límite de fase es un proceso que incluye la reacción propiamente dicha. Aunque la ecuación parabólica de velocidad de reacción parte de premisas que no se dan en la realidad, se ha estudiado la validez de la ecuación parabólica de velocidad de Jander (29) para la reacción [2] de formación de $\mathrm{B}_{-} \mathrm{C}_{2} \mathrm{~S}$, así como para el proceso global de formación de wollastonita, que resulta de combinar las ecuaciones [2] y [3], y que podemos 
escribir como sigue:

$$
\mathrm{CaO}+\mathrm{SiO}_{2} \rightarrow \mathrm{CaSiO}_{3}[4]
$$

La ecuación parabólica de velocidad de Jander resulta ser:

$$
F(\gamma)=\left[1-(1-x)^{\frac{1}{3}}\right]=2 \frac{K D t}{r_{0}^{2}}=K_{J} t
$$

donde:

$\mathrm{D}$ = coeficiente de difusión promedio del $\mathrm{CaO}$ a través de la capa de silicato cálcico.

$\mathrm{k}=$ constante adimensional.

$\mathrm{r}_{0}=$ radio inicial del grano de sílice.

$\mathrm{K}_{\mathrm{j}}=$ constante de velocidad de reacción.

$\mathrm{g}$ = extensión de la reacción para distintos valores del tiempo. Se puede determinar en función de cualquier variable relacionada con la concentración de una determinada fase (masa, intensidad, etc.). Este parámetro se expresa mediante la siguiente ecuación

$$
\gamma=\frac{m_{1}-m_{0}}{m_{0}}
$$

siendo:

$\mathrm{m}_{0}=$ masa inicial de la fase considerada.

$\mathrm{m}_{1}=$ masa de la fase considerada en cualquier momento de la reacción.

Para comprobar si las reacciones en estado sólido descritas anteriormente se ajustan a la ley parabólica de velocidad de Jander, se ha aplicado dicha ley a las ecuaciones [2] y [4]. En ambos casos se comprobó que la fase inicial del proceso podía ser descrita por la ecuación de Jander, y así la Fig. 5, muestra relación lineal en las primeras etapas del mismo, aunque posteriormente, según puede comprobarse en esta figura, la reacción avanza más lentamente, y ya no es aplicable la ecuación de Jander.

En la Tabla IV se muestran los valores de la constante de velocidad de la reacción de formación del $\beta_{-} C_{2} S$ según el modelo tratado. Debe tenerse en cuenta que el cálculo de $\mathrm{K}_{\mathrm{J}}$ para la formación del $\mathrm{B}_{-} \mathrm{C}_{2} \mathrm{~S}$ es poco fiable, ya que este en algunas muestras desaparece en las primeras etapas de la reacción. Sin embargo, aunque los valores de $\mathrm{K}_{\mathrm{J}}$ obtenidos para la fase $\mathrm{B}_{\mathrm{B}} \mathrm{C}_{2} \mathrm{~S}$ son poco precisos, pueden ser usados en sentido relativo y permiten establecer comparaciones entre las distintas muestras. Los valores de esta tabla, confirman la secuencia de aparición del $\mathrm{B}_{-} \mathrm{C}_{2} \mathrm{~S}$, anteriormente apuntada:

$$
\mathrm{DS}>\mathrm{BS}>\mathrm{AS}>\mathrm{CS}
$$

En la Fig. 6, se ha representado la constante de velocidad $\mathrm{K}_{\mathrm{J}}$ de la reacción de formación del $B-C_{2} S$ frente a la superficie específica de las materias primas que aportan sílice. Puede verse la existencia de una cierta correlación lineal entre ambos parámetros. Sin embargo, la formación de wollastonita no aumenta con la superficie específica de la materia prima silícea. Por el contrario, cuanto mayor es ésta, la desaparición del $\mathrm{B}_{3} \mathrm{C}_{2} \mathrm{~S}$ para formar wollastonita es tanto más lenta. Así, en muestras como la DS hay una gran estabilización del $\mathrm{B}_{-} \mathrm{C}_{2} \mathrm{~S}$, que se manifiesta porque la proporción de wollastonita forma-
TABLA IV.

CONSTANTES DE VELOCIDAD DE LA REACCiÓN DE FORMACIÓN DE $\beta_{-} \mathrm{C}_{2} \mathrm{~S}$ SEGÚN EL MODELO DE JANDER

\begin{tabular}{|c|c|c|}
\hline Composición & $\begin{array}{c}\mathrm{K}_{\mathrm{J}}\left(\mathrm{B}-\mathrm{C}_{2} \mathrm{~S}\right) \times 10^{3} \\
\left(\mathrm{~min}^{-1)}\right.\end{array}$ & $\begin{array}{c}\text { Intervalo de medida } \\
(\min .)\end{array}$ \\
\hline AS & 7,9 & 10 \\
\hline BS & 15,7 & 10 \\
\hline CS & 1,1 & 300 \\
\hline DS & 91,3 & 10 \\
\hline
\end{tabular}

TABLA V.

CONSTANTES DE VELOCIDAD DE REACCIÓN DE FORMACIÓN DE WOLLASTONITA SEGÚN EL MODELO DE JANDER

\begin{tabular}{|c|c|c|}
\hline Composition & $\begin{array}{c}\mathrm{K}_{\mathrm{J}}(\mathrm{Wo}) \times 10^{3} \\
\mathrm{~min}^{-1}\end{array}$ & Intervalo de medida \\
\hline AS & 8,7 & 60 \\
\hline BS & 5,9 & 60 \\
\hline CS & 0,5 & 420 \\
\hline DS & 3,4 & 60 \\
\hline
\end{tabular}

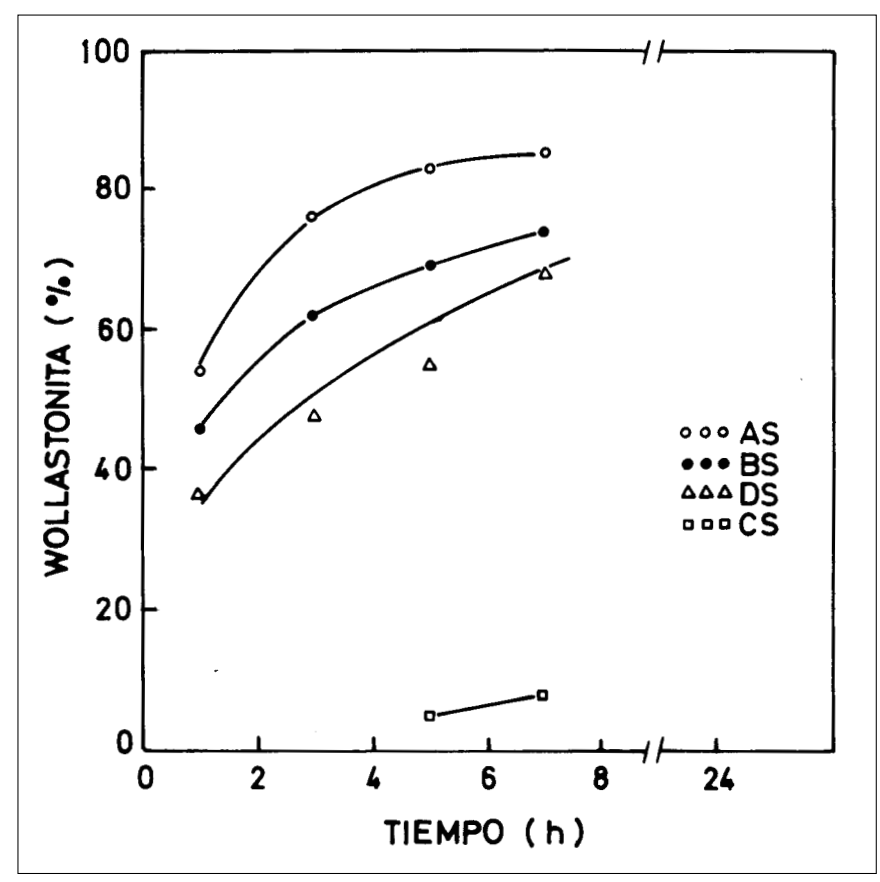

Fig. 4.- Variación del contenido de wollastonita de las composiciones con el tiempo de tratamiento 
TABLA VI.

VALORES DE $m$ PARA MODELOS DE CRECIMIENTO DE NÚCLEOS

\begin{tabular}{|c|c|c|}
\hline Condiciones de frontera & Control por interfase & Control por difusión \\
\hline \multicolumn{2}{|c|}{ Crecimiento tridimensional } & sferas \\
\hline Veloc. de nucleación constante & 4 & 2,5 \\
\hline Veloc. de nucleación nula & 3 & 1,5 \\
\hline Veloc. de nucleación decreciente & $3-4$ & $1,5-2,5$ \\
\hline \multicolumn{2}{|c|}{ Crecimiento bidimensional } & Placas \\
\hline Veloc. de nucleación constante & 3 & 2 \\
\hline Veloc. de nucleación nula & 2 & 1 \\
\hline Veloc. de nucleación decreciente & $2-3$ & $1-2$ \\
\hline \multicolumn{2}{|c|}{ Crecimiento unidimensional } & Cilindros \\
\hline Veloc. de nucleación constante & 2 & 1,5 \\
\hline Veloc. de nucleación nula & 1 & 0,5 \\
\hline Veloc. de nucleación decreciente & $1-2$ & $0,5-1,5$ \\
\hline
\end{tabular}
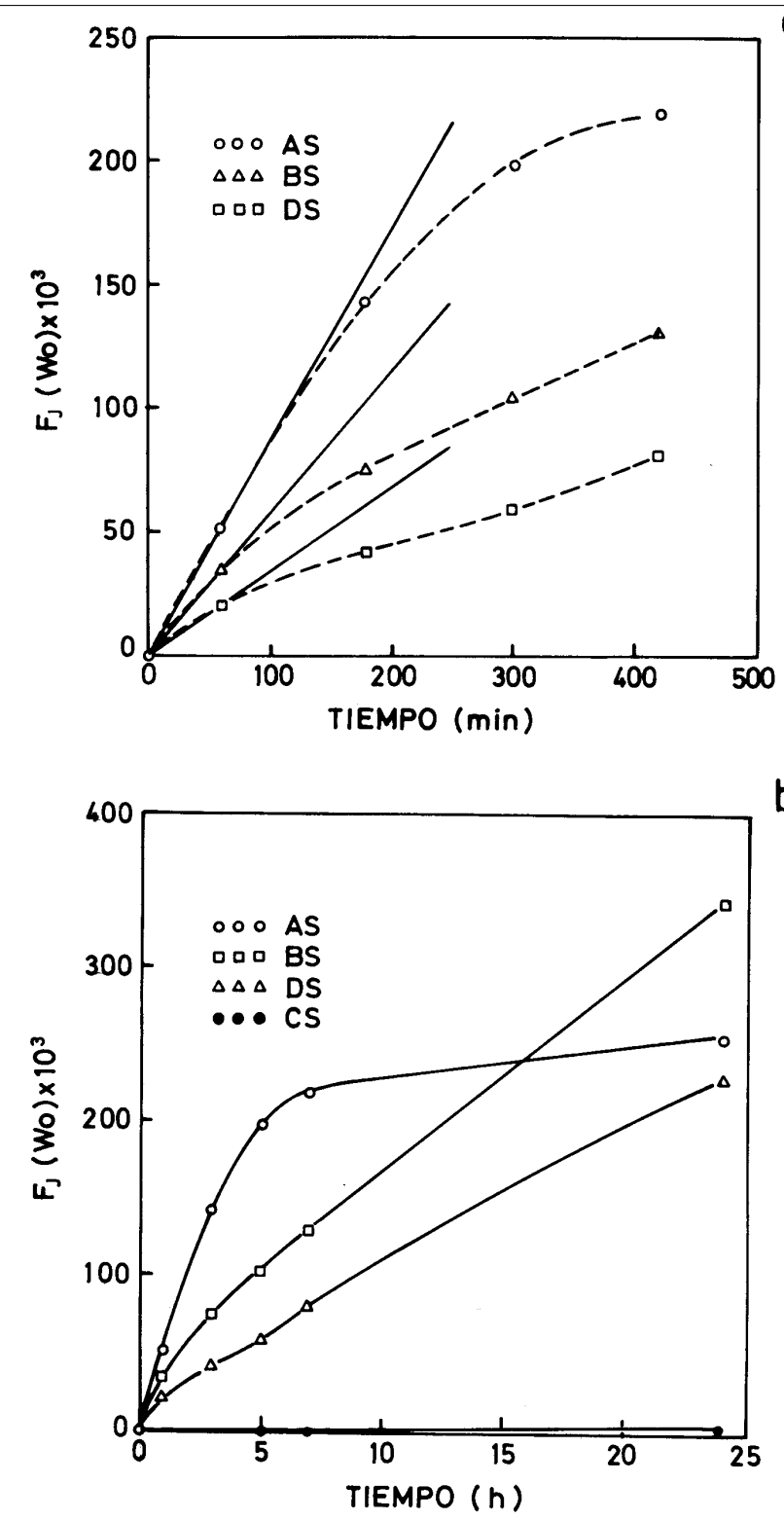

Fig. 5.- Función de Jander para el proceso global de formación de wollastonita: a) en la etapa inicial; b) a lo largo de todo el proceso da aumenta muy lentamente.

En la Tabla V se muestran los valores de la constante de velocidad para el proceso global de formación de wollastonita, tanto en la etapa inicial, como en el proceso global de formación de wollastonita. Estos resultados concuerdan con los de Packter y Zaidi quienes han estudiado la cinética y el mecanismo de reacción de las reacciones heterogéneas entre $\mathrm{CaO}$ y $\mathrm{SiO}_{2}$ (13), y entre $\mathrm{CaO}$ y $\mathrm{Al}_{2} \mathrm{O}_{3}$ a distintas temperaturas y en presencia de cloruros de litio, sodio o calcio como mineralizadores (14). En todos los casos se observó, que la etapa inicial de la reacción, hasta llegarse a aproximadamente a un desarrollo de un $60 \%$ de la misma podía ser descrita por la relación de Jander, es decir, la velocidad de reacción esta inicialmente controlada por la difusión. También Weisweiler y col (30) han investigado las reacciones que tienen lugar entre interfases planas $\mathrm{CaO} / \mathrm{SiO}_{2}$, comprobando que el crecimiento de las zonas de reacción sigue la ley parabólica de velocidad de reacción para las reacciones controladas por difusión. Más recientemente Morais et al. (31), han presentado algunos resultados preliminares sobre la cinética de formación de wollastonita por reacción en estado sólido, deduciendo que era válido el modelo de Jander, y que se producía difusión de iones $\mathrm{Ca}^{2+}$ en la sílice. Sin embargo como puede apreciarse en la figura 5 (b) la reacción ya no se ajusta a la ecuación de Jander, debido a que la reacción avanza más lentamente por tener que difundirse las partículas de $\mathrm{CaO}$ a través de una capa de producto de espesor creciente para alcanzar las partículas de sílice. En estas condiciones la velocidad de reacción depende del coeficiente de difusión del $\mathrm{CaO}$ a través de la capa del producto de reacción, y como se ha indicado anteriormente, no puede aplicarse al proceso global de formación de wollastonita la ecuación de velocidad de Jander. Los modelos para las reacciones en estado sólido entre compactos en polvo se basan en la consideración de que inicialmente la difusión en superficie cubre la superficie de la partícula reaccionante con una capa continua

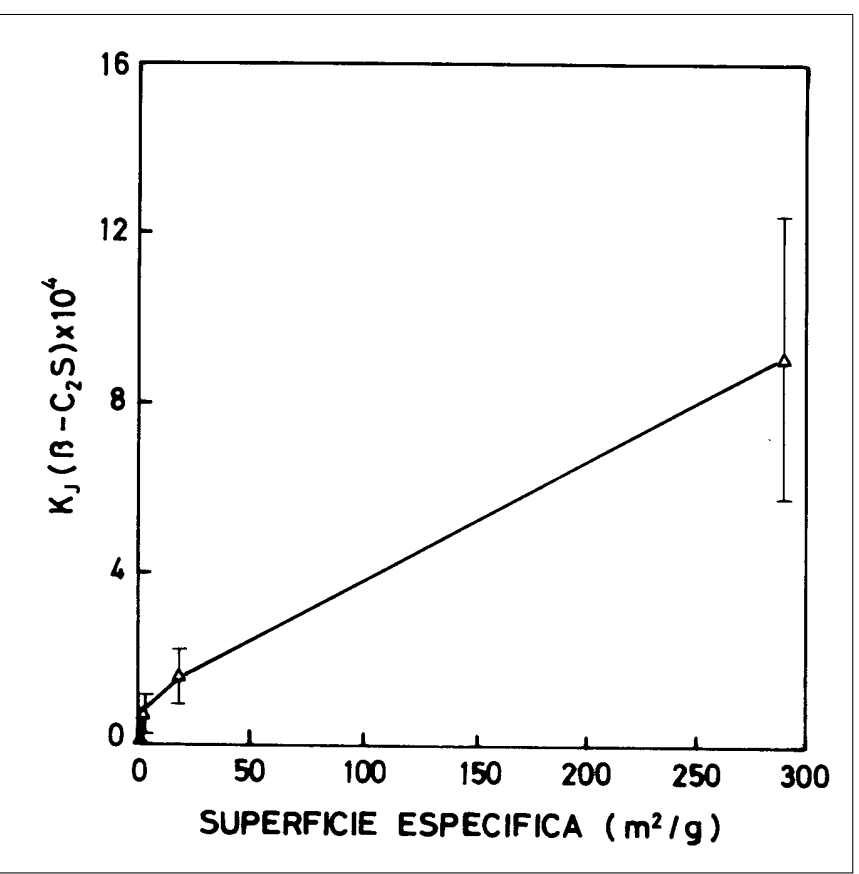

Fig. 6.- Relación entre la constante de Jander para la reacción de formación de $\beta-C_{2} S$ y la superficie específica de las materias primas silíceas. 
de producto. Realmente, no hay una velocidad de reacción concreta, ya que $K_{\mathrm{J}}$ disminuye a medida que aumenta la capa de producto. Por ello se ha analizado otro modelo de alcance más general.

\section{ii) Modelo de Avrami}

Hay otra forma de analizar, tanto la formación inicial del producto como su crecimiento. Aquella considera la nucleación de los productos en sitios activos y la velocidad a la que las partículas nucleadas crecen. Se trata en definitiva de una reacción de tipo aditivo entre las sustancias A y B donde la fase producto crece a partir de núcleos distribuidos al azar dentro de la fase reactiva A. Todas las ecuaciones basadas en crecimiento de núcleos tienen la siguiente forma general de acuerdo con Hulbert (32):

$$
\ln \frac{1}{1-x}=K_{A} t^{m}
$$

donde $m$ es un parámetro que depende del mecanismo de la reacción, de la velocidad de nucleación y de la geometría de los núcleos. Los valores de $\mathrm{m}$ y su significado han sido resumidos por Hulbert (32) y, se encuentran en la Tabla VI. En general el valor de $\mathrm{m}$ aumenta cuando el crecimiento tiene lugar a lo largo de dos o tres dimensiones y es mayor para los procesos controlados por la interfase que para los procesos controlados por difusión.

Si se toman logaritmos en la ecuación anterior, se tendrá:

$$
\ln \left(\ln \frac{1}{1-x}\right)=\ln K_{A}+m \ln t
$$

Esto significa que en una reacción en estado sólido que pueda ser explicada por un modelo de crecimiento de núcleos, la representación de $\ln [\ln (1 / 1-x)]$ frente a $\operatorname{lnt}$ debe dar una línea recta de pendiente $\mathrm{m}$ y ordenada en el origen $\ln \mathrm{K}_{\mathrm{A}}$. Esto es lo que se aprecia claramente en la Fig. 7, donde se muestra el resultado de la aplicación del modelo de Avrami, tanto para la evolución del ß-silicato dicálcico [Fig. 7 (a)] como para la formación de wollastonita [Fig. 7 (b)]. La gráfica referida al proceso global de formación de wollastonita [Fig.7 (b)] muestra tres rectas de pendiente prácticamente igual para las composiciones AS, BS y DS, y una pendiente más pronunciada para la composición CS, basada en cuarzo, lo que parece ser consecuencia de un mecanismo de reacción diferente. En todos los casos, los valores de $m$ son tan bajos que corresponden a procesos controlados principalmente por difusión.

Es decir, como puede apreciarse en la Fig. 7, el modelo de Avrami explica bien tanto la cinética de la reacción de formación de $\mathrm{B}_{-} \mathrm{C}_{2} \mathrm{~S}$, como la de wollastonita. En la Tabla VII, se muestra la constante de reacción de formación de wollastonita, así como los correspondientes valores de m. No son comparables los valores de $\mathrm{K}_{\mathrm{J}}$ (Tabla V) y de $\mathrm{K}_{\mathrm{A}}$ (Tabla VII), ya que corresponden a procesos diferentes. Así, mientras $K_{\mathrm{J}}$ cambia con el tiempo de reacción, $\mathrm{K}_{\mathrm{A}}$ es independiente del mismo, como se desprende de la Tabla VIII.
TABLA VII.

CONSTANTE DE VELOCIDAD DE REACCIÓN GLOBAL DE FORMACIÓN DE WOLLASTONITA SEGÚN EL MODELO DE AVRAMI

\begin{tabular}{||c|c|c|c|}
\hline \hline Composición & $\mathrm{m}$ & $\mathrm{K}_{\mathrm{A}}(\mathrm{Wo}) \times 10^{2}$ & $\mathrm{r}^{2}(*)$ \\
\hline AS & 0,47 & 11,77 & 98 \\
\hline BS & 0,46 & 9,26 & 99 \\
\hline CS & 0,96 & 2,10 & 99 \\
\hline DS & 0,47 & 6,39 & 97 \\
\hline
\end{tabular}

$(*)$ Coeficiente de determinación del ajuste de los puntos a una recta.

TABLA VIII.

\begin{tabular}{|c|c|c|c|c|}
\hline Composición & Tiempo (min.) & $\ln \mathrm{K}_{\mathrm{A}}$ & $\begin{array}{c}\text { Valor } \\
\text { promedio }\end{array}$ & $\sigma(* *)$ \\
\hline AS & $\begin{array}{c}60 \\
180 \\
300 \\
420 \\
\end{array}$ & $\begin{array}{l}-2,18 \\
-2,09 \\
-2,12 \\
-2,20\end{array}$ & $-2,15$ & 0,045 \\
\hline BS & $\begin{array}{c}60 \\
180 \\
300 \\
420\end{array}$ & $\begin{array}{l}-2,35 \\
-2,40 \\
-2,44 \\
-2,45\end{array}$ & $-2,41$ & 0,039 \\
\hline CS & $\begin{array}{c}300 \\
420 \\
1.440\end{array}$ & $\begin{array}{l}-8,52 \\
-8,37 \\
-8,47\end{array}$ & $-8,45$ & 0,065 \\
\hline DS & $\begin{array}{c}60 \\
180 \\
300 \\
420\end{array}$ & $\begin{array}{l}-2,67 \\
-2,84 \\
-2,88 \\
-2,68\end{array}$ & $-2,78$ & 0,093 \\
\hline
\end{tabular}

VALORES DE $\left.\ln \mathrm{K}_{\mathrm{A}}{ }^{*}{ }^{*}\right)$ DETERMINADOS A Distintos TIEMPOS DE REACCIÓN.

$\left({ }^{*}\right) \mathrm{K}_{\mathrm{A}}=$ Constante de velocidad de la reacción de formación de wollastonita según el modelo de Avrami

$\left.{ }^{* *}\right) \sigma=$ Desviación estándar de los valores de $\ln \mathrm{K}_{\mathrm{A}}$

\section{CONCLUSIONES}

Del estudio realizado se deducen las siguientes conclusiones:

1. La primera fase de reacción observada en todos los casos fue el $\mathrm{B}-\mathrm{C}_{2} \mathrm{~S}$.

2. La velocidad de formación del ß-silicato dicálcico sigue la secuencia:

$$
\mathrm{DS}>\mathrm{BS}>\mathrm{AS}>\mathrm{CS}
$$

3. Se considera que el $\mathrm{B}-\mathrm{C}_{2} \mathrm{~S}$ puede quedar estabilizado por la aparición de una matriz vítrea alrededor de las partículas de $\mathrm{SiO}_{2}$, lo cual impide su transformación en la fase $\gamma$, mucho más voluminosa, siendo esta estabilización mayor a medida que aumenta la superficie específica de la materia prima silícea.

4. La velocidad de formación de wollastonita sigue la siguiente secuencia:

$$
\mathrm{AS}>\mathrm{BS}>\mathrm{DS}>\mathrm{CS}
$$

Esta secuencia difiere de la de formación del $\mathrm{B}_{-} \mathrm{C}_{2} \mathrm{~S}$, a causa de la estabilización de éste.

5. El proceso global de formación de wollastonita se ajusta en las etapas iniciales a la ecuación parabólica de velocidad de Jander. Posteriormente, la difusión del calcio tiene lugar a través de una capa de espesor creciente, y el modelo de Jander ya no es aplicable.

6. La reaccion de formación de wollastonita se ajusta al mode- 
lo de Avrami, basado en la nucleación de los productos en sitios activos. La aplicación de este modelo permite concluir que ambos procesos están controlados por difusión.

\section{AGRADECIMIENTO}

Los autores expresan su agradecimiento a D. Jose Manuel Cordero por su colabaración en este trabajo.
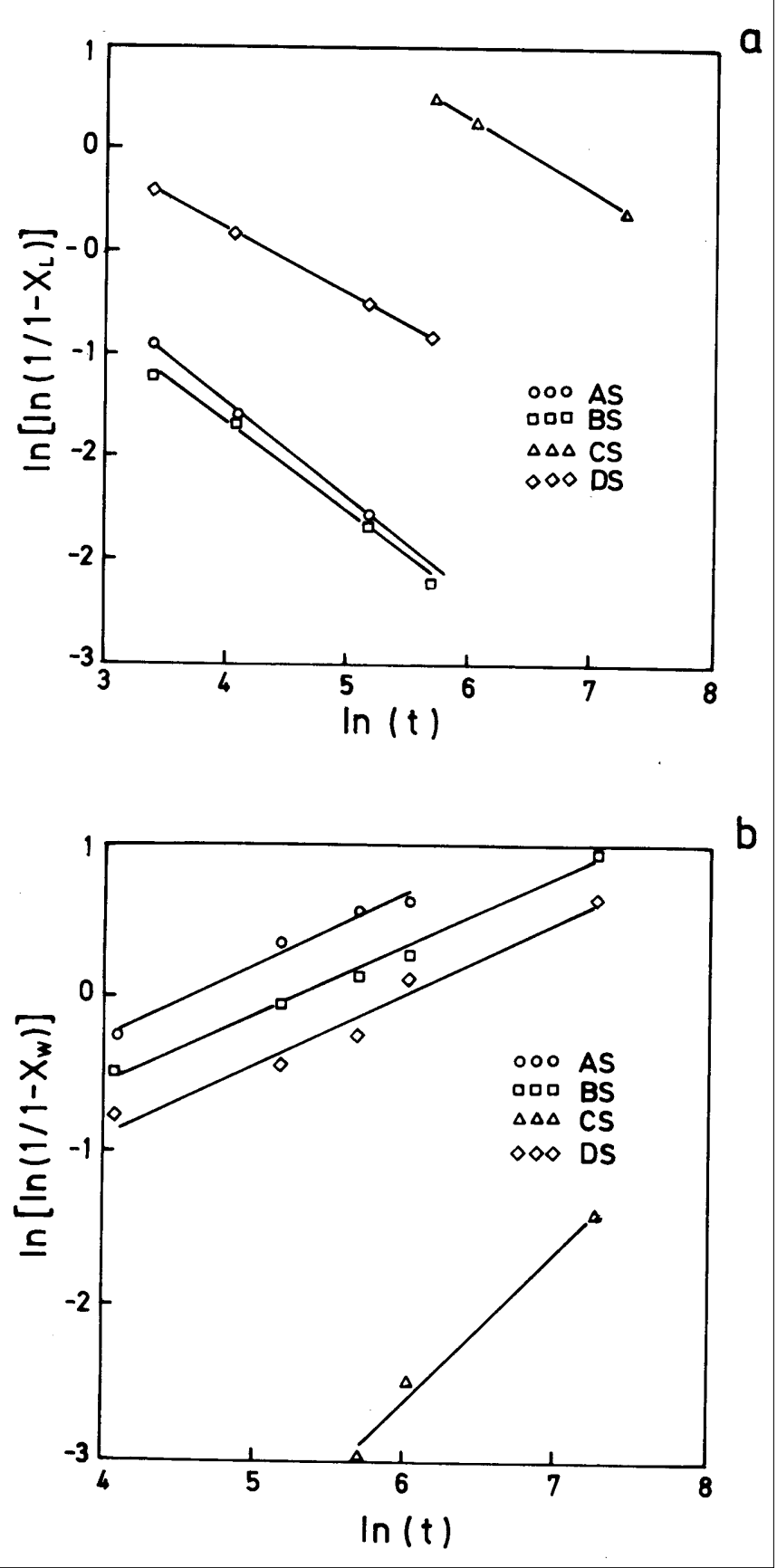

Fig. 7.- Representación de $\ln (\ln 1 / 1-x)$ frente a lnt: a) para la reacción de formación de $\mathrm{B}-\mathrm{C}_{2} \mathrm{~S} ; \mathrm{b}$ ) para el proceso global de formación de wollastonita

\section{BIBLIOGRAFÍA}

1. J.M. González Peña, F. Sandoval, J.E. Enrique, V. Beltrán. “Utilización de materias primas de bajo coste en la obtención de wollastonita". Proc. I Congreso-Exposición Argentino y II Iberoamericano de Cerámica, 9-16, Vidrio y Refractarios. Edit. Asociación Argentina de Cerámica, Buenos Aires (1988).

2. H.G. Kurczyk, J. Wührer. "Synthetic wollastonite and its use in ceramic bodies". Interceram, 20, [2], 119-125 (1971).

3. K.C. Rieger. “Wollastonite”. Amer. Ceram. Soc. Bull., 76, [6], 139-140 (1997).

4. Duda, R. y Rejl, L. Minerals of the world. Asch Cape Press, New York, 1990).

5. Roskill Information Services. The Economics of Wollastonite, 1996. .The Minerals Metals \& Materials Society, Diciembre, 1996.

6. H.G. Kurczyk. "Synthetic diopside and synthetic wollastonite-new raw materials for.ceramics". Proc. $3^{\text {rd }}$ Int. Meet. on Modern Ceram. Tech., 22-31, Rimini (Italia), 27-31 de Mayo (1975).

7. I. Kotsis, A. Ballogh. "Synthesis of wollastonite". Ceram. Int., 15, [2], 79-85 (1989).

8. A. Ibáñez, F. Sandoval: "Wollastonita: propiedades, síntesis y aplicaciones cerámicas”. Bol. Soc. Esp. Cerám. y Vidr., 32, [6], 349-361 (1993).

9. H. Schmalzried, "Solid state reaction". Treatise of solid state chemistry, vol. 4, 266-268, Edit. N.B. Hannay, Plenum Press New York (1976).

10. M.K. Gal'perina, O.S. Grum Gzhimailo, V.S. Mitrokin, N.P. Tarantul. "Synthesizing wollastonite from tripoli". Steklo i Keram., 39, [2], 16-17 (1982).

11. F. Kupka. "Wollastonite synthesis". Sb. Geol. Ved., 19 159-185 (1984).

12. A. Berezky. "Relationskinetik des Systems $\mathrm{CaO}-\mathrm{SiO}_{2}$ und $\mathrm{CaO} \mathrm{Al}_{2} \mathrm{O}_{3}$ in amwesenheit von Katalisatoren". Silikattechnik, 11, [10], 474-477 (1960).

13. A. Packter, S.M.A. Zaidi. "Mineralisatorwirkungvon Lithium und Kalciumchlorid bei der Bildung von B-Dikalciumsilikat". Silikattechnik, 26, [1], 14-16 (1976).

14. N.V. Brovkova. "Using beneficiated wollastonite for wall tile". Steklo i Keram, 36, [11], 15-16 (1979).

15. A. Packter, S.A. Zaidi. "Alumina-calcium oxide reactions with metal chlorides as mineralizers". Silicat. Indus., LV, [3-4], 95-98 (1990).

16. V. Andreeva, G. Fekeldiev. "Use of non-conventional raw materials in ceramic production". Ceram. Int., 12, 229-235 (1986)

17. M.I. Ryschenko, V.A. Shekhotshava, G.A. Salditire, V.I. Zukovin, A.X. Belik, V.V. Gutarova. "Use of secondary soda production materials in wollastonite synthesis". Steklo i Keram., 45, [10], 6-8 (1988).

18. A. Ibáñez, J.M Gonzalez Peña, F. Sandoval. "Solid state reaction for producing B-wollastonite". Amer. Ceram. Soc. Bull., 69, [3], 374-378 (1990).

19. D.B. Lucas, P. Santos. "Synthesis of wollastonite at low temperature from low cost raw materials". Proc. Europ. Ceram. Soc. $3^{\text {rd }}$ Conf., vol. 2., 941-946. Edit. P. Durán, J.F. Fernández, Public. Faenza Editrice Iberica, S.L., Madrid (1993).

20. C.J. Chang y W.M. Kriven. "Physical stabilization of the $B 6 \mathrm{~g}$ transformation in dicalcium silicate". J. Am. Ceram. Soc., 75, [6], 1621-1627 (1992).

21. E.L. Calacal, O.J. Whittmore. "The sintering of diatomite". Amer. Ceram. Soc. Bull., 66, [5], 790-793 (1987).

22. J. F. Lemmons, “Diatomite”. Amer. Ceram. Soc. Bull.,76, [6],94-95 (1997).

23. F.L. Kadey. "Diatomite". Industrial Minerals and Rocks, 5.0 edic. Society of Mining Engineers, Nueva York (1983).

24. F.H. Chung. "Quantitative interpretation of X-ray diffraction patterns of mixtures. I. Matrix flushing method for quantitqtive multicomponent analysis". J. Appl. Crystall, vol 7, Parte 6, 12, 519-525 (1974).

25. H. Midgley. "The polymorphim of calcium orthosilicate". Proc. Int. Cong. Chem. Cem., $6^{\text {th }}$, Suppl., 1-16 (1974).

26. K. Nielsen. "The Importance of the $\mathrm{a}={ }_{\mathrm{L}}-\mathrm{a}={ }_{\mathrm{H}}$ Transition in the Polymorphism of Dicalcium Silicate". Silic. Ind., 37 [5] 136-138 (1972).

27. C. Remy, D. Anndrault, M. Madon. High-Temperature, High-Pressure X-Ray Investigation of dicalcium Silicate. J. Am. Ceram. Soc. 80 [4] 851-860 (1997).

28. A.R. West. "Solid state chemistry and its applications". Ed. John Wiley and Sons, Chichester-New York-Brisbane-Toronto-Singapore, (1985).

29. W. Jander. "Reactions in solid state at high temperatures. I". Z. Anorg. Chem., 163, [1-2], 1-8 (1927).

30. W. Weisweiler; E. Osen y J. Eck. "Kinetic studies in the $\mathrm{CaO}-\mathrm{SiO}_{2}$ system. Part I. Mechanism and kinetics data of the reactions between $\mathrm{CaO}$ and $\mathrm{SiO}_{2}$ powder compacts". Cement Concr. Res. 16, 283-295 (1986).

31. L. Morais, A. Correia y T. Fonseca. "Kinetic treatment of the solid state of wollastonite". Proc. Europ. Ceram. Soc. 3rd. Conf., vol, 342-347. Ed. P. Duran y J.F. Fernández. Public. por Faenza Editrice Ibérica, S.L., Madrid (1993).

32. S.F. Hulbert. "Models for solid-state reactions in powdered compacts: A review".

J. Brit. Ceram. Soc., 5, 11-20 (1969).

Recibido: 15-10-96

Aceptado: 1-4-98 